\title{
SARS-CoV-2 testing in infertile patients: different recommendations in Europe and America
}

\author{
Antonio La Marca ${ }^{1,2,3}$ (I) $\cdot$ Scott M Nelson ${ }^{4,5,6}$ \\ Received: 3 June 2020 / Accepted: 9 July 2020 / Published online: 17 July 2020 \\ (C) Springer Science+Business Media, LLC, part of Springer Nature 2020, corrected publication 2020
}

\begin{abstract}
The incorporation of severe acute respiratory syndrome coronavirus 2 (SARS-CoV-2) testing into patient care algorithms has been proposed to mitigate risk. However, the two main professional societies for human reproduction (ESHRE and ASRM) appear divergent on their clinical utility and whether they should be adopted. In this opinion paper, we review the currently available tests and discuss the strengths and weaknesses of the proposed clinical care pathways. Nucleic acid amplification tests are the cornerstone of SARS-CoV-2 testing but test results are largely influenced by viral load, sample site, specimen collection method, and specimen shipment technique, such that a negative result in a symptomatic patient cannot be relied upon. Serological assays for SARS-CoV-2 antibodies exhibit a temporal increase in sensitivity and specificity after symptom onset irrespective of the assay used, with sensitivity estimates ranging from 0 to $50 \%$ with the first 3 days of symptoms, to 83 to $88 \%$ at 10 days, increasing to almost $100 \%$ at $\geq 14$ days. These inherent constraints in diagnostics would suggest that at present there is inadequate evidence to utilize SARS-CoV-2 testing to stratify fertility patients and reliably inform clinical decision-making. The failure to appreciate the characteristics and limitations of the diagnostic tests may lead to disastrous consequences for the patient and the multidisciplinary team looking after them.
\end{abstract}

Keywords COVID-19 · SARS-CoV-2 · ESHRE · ASRM · IVF

\section{Introduction}

There are principally two types of tests available for severe acute respiratory syndrome coronavirus 2 (SARS-CoV-2) and its associated disease coronavirus disease 2019 (COVID-19): viral tests and antibody tests. The viral assays are direct tests as they are designed to detect the virus and therefore reflect the current infection. In contrast, the antibody assays are indirect tests, as they do not detect the virus, but rather ascertain

Antonio La Marca

antlamarca@libero.it; antonio.lamarca@unimore.it

1 Department of Medical and Surgical Sciences for Children and Adults, University of Modena and Reggio Emilia, Modena, Italy

2 Clinica Eugin, Modena, Italy

3 Obstetrics \& Gynecology, University Hospital Modena, Modena, Italy

4 School of Medicine, University of Glasgow, Glasgow, UK

5 NIHR Bristol Biomedical Research Centre, Bristol, UK

6 The Fertility Partnership, Oxford, UK established seroconversion to previous infection, or early seroconversion to ongoing infection.

The utilization of direct molecular diagnostic testing based on sequencing of the SARS-CoV-2 RNA genome has been critical in identifying infected individuals in the early phase of the disease. Antibody testing may be relevant for other clinical applications including (i) diagnosis and triage of patients who seek medical attention in the later phases of the disease; (ii) contact tracing; (iii) stratifying workforces and patients if immunity is shown to be lasting; and (iv) sero-epidemiological studies to understand the extent of COVID-19 spread.

To be able to recommend to clinicians the most appropriate clinical conduct in the management of patients who are triage positive, hence at risk of being infected, or of patients that may present some symptoms during the cycle of treatment, we must understand the main laboratory characteristics of the diagnostic tests available at the moment.

\section{Tests for direct detection of SAR-CoV-2}

The recommended diagnostic test for SARS-CoV-2 infection is by viral nucleic acid detection by nucleic acid amplification 
tests (NAAT), such as RT-PCR. The current database held by the FIND presently contains 275 commercialized molecular assays with many others in the development pipeline (search performed on May 14, 2020). There is now a huge amount of experience with these tests and widespread recognition of their limitations. Specifically, test results are largely influenced by viral load, sample site, and method of specimen collection and specimen shipment technique, all of which can contribute to a false-negative result. Of these viral load and sampling sites are the most variable, with the viral load in nasopharyngeal swabs at its highest at the time of symptom onset and decreasing monotonically thereafter [1,2]. Analysis of viral temporal dynamics suggests that viral shedding may begin 2 to 3 days before the appearance of the first symptoms, facilitating pre-symptomatic or asymptomatic transmission [3]. Given all these sources of variability, a negative test resulting from respiratory samples does not exclude the possibility of SARS-CoV-2, and if symptomatic they should be managed as a positive case and resampling undertaken.

Antigen detection tests are designed to directly detect viral particles in biological samples like nasopharyngeal secretions. Many rapid antigen tests have been proposed; however, their performance would appear limited. Despite initial suggestions of positive predictive values as high as $100 \%$, and negative predictive values for $97 \%$ for patients with high viral load, the negative predictive value falls away steeply with reducing viral load, with as low as $32 \%$ reported in cases with a low viral load [4]. Hence, the principal concern for this kind of test is the false-negative rate due to either a low or variable viral load and the variability in sampling. Previous experience with influenza antigen testing which is known to have low sensitivity further highlights the need for caution, as a low falsenegative rate will be critical to ensure that appropriate public health measures are not omitted [5]. Despite these limitations, given their rapidity and ability to be deployed to any setting, continued efforts towards improving this technology are worthwhile.

\section{Serological testing-for evidence of SARS-CoV-2 exposure}

The temporal dynamics of seroconversion after SARS-CoV-2 exposure have been delineated in conjunction with specific antibody immunoassay development. The data would suggest that there is either a simultaneous or sequential seroconversion for both IgM and IgG titers, with titers potentially plateauing within 6 days after seroconversion [6]. In a series of sixty-three patients with prolonged follow-up, overall seroconversion before hospital discharge was $96.8 \%$ [7]. This and other temporal studies have highlighted the chronological nature of testing and the critical need to account for the time of sampling in test interpretation. For example in a cohort of 173 patients with confirmed
SARS-CoV-2 infection, in the early phase of illness (within 7day since onset), the NAAT exhibited $66.7 \%$ sensitivity and only $38.3 \%$ of the cohort had evidence of antibodies [8]. Further evidence of the temporal nature of conversion was observed with the more advanced techniques for immunoglobulin detection, such as the automated chemiluminescent assay [9]. A recent systematic review and meta-analysis of antibody test performance derived from 54 study cohorts with 15,976 samples reported that the combination of $\mathrm{IgG} / \mathrm{IgM}$ had a sensitivity of $30.1 \%$ (95\% CI 21.4 to 40.7 ) for 1 to 7 days, $72.2 \%$ (95\% CI 63.5 to 79.5 ) for 8 to 14 days, and $91.4 \%$ (95\% CI 87.0 to 94.4 ) for 15 to 21 days [6]. Estimates of accuracy beyond three weeks were based on smaller sample sizes and fewer studies, with $96.0 \%$ (95\% CI 90.6 to 98.3 ) sensitivity for 21 to 35 days. The authors noted that there were insufficient studies to estimate the sensitivity of tests beyond 35 days of post-symptom onset [6]. Notably, summary specificities derived from 35 studies exceeded $98 \%$ for all target antibodies [6]. Collectively this data suggests that there is no role for antibody testing in the diagnosis of the disease in its very early phase, with the combined use of NAAT and Ab tests markedly improving the sensitivities of pathogenic-diagnosis for COVID-19 patients in the different phases of their infection journey [10].

At present an extensive range of serological assays continue to be released; however, few have undergone external validation. Gonzalez and colleagues reviewed four web databases for SARS-CoV-2 immunoassays and by April 4, 2020, there were already 226 immunoassays from 20 different countries. The technical data sheet was available online in only $22 \%$ of tests; 23 claimed regulatory certification and only four had PubMed listed papers [11]. With this overwhelming range of possibilities, the selection of which test to adopt is problematic. Pragmatically we would suggest using an automated and scalable immunoassay produced by a well-known established manufacturer, with a complete and clear technical data sheet, a regulatory certification issued by a health authority, and evidence of independent validation. Table 1 summarizes the main performance characteristics of the commonest used assays relative to the stage of the disease, with the overall strengths and weaknesses of serological testing summarized in Table 2.

Rapid serological assays which are similar in design to the lateral flow immunoassays used for pregnancy tests have also been developed for the rapid detection of SARS-CoV-2 antibodies. These tests would enable point-of-care detection of SARS-CoV-2 exposed subjects without the necessary laboratory infrastructure or transport framework required for centralized testing. As may be anticipated, the rapid assays have a low diagnostic performance when compared with the ELISA assays, reflecting the well-known technical differences between the two methodologies but also possible low antibody concentrations. The evaluation of six POC tests in a mix of 110 cases of COVID-19, other coronaviruses, other viruses, and negative controls revealed sensitivities ranging from 80 to 


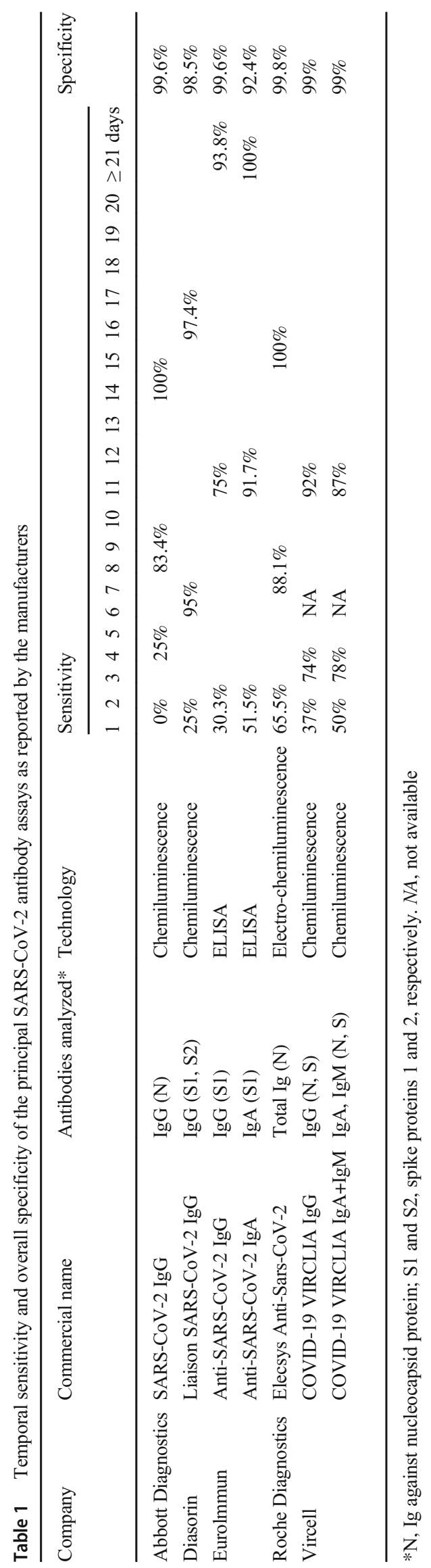


Table 2 Strengths and limitations of antibody testing for diagnosing SARS-CoV-2

\begin{tabular}{|c|c|}
\hline Strengths & Limitations \\
\hline $\begin{array}{l}\text { Possible identification of pauci-symptomatic and re- } \\
\text { covered subjects }\end{array}$ & Inter-person variability in seroconversion \\
\hline $\begin{array}{l}\text { Good diagnostic performance after } 7-10 \text { days from } \\
\text { the onset of symptoms (lower for the rapid assays) }\end{array}$ & $\begin{array}{l}\text { False-negative tests are possible and pose a challenge } \\
\text { particularly due to temporal nature of seroconversion }\end{array}$ \\
\hline Easy and safe administration & $\begin{array}{l}\text { Lack of independent validation of available tests in } \\
\text { immunocompromised populations }\end{array}$ \\
\hline Cheap and short time to results & Rapid tests have low sensitivity \\
\hline $\begin{array}{l}\text { Possible correlation of antibody titers with the } \\
\text { severity of the syndrome }\end{array}$ & $\begin{array}{l}\text { Scarce information on the persistence of antibodies } \\
\text { after the first infection }\end{array}$ \\
\hline Useful for vaccine seroconversion confirmation & $\begin{array}{l}\text { Very scarce information on the strength and long-term } \\
\text { implication of immunity }\end{array}$ \\
\hline $\begin{array}{l}\text { Improve the detection of COVID-19 positive rate in } \\
\text { suspected subjects when combined with NAAT }\end{array}$ & Lack of robust diagnostic accuracy studies \\
\hline $\begin{array}{l}\text { Easy monitoring of healthy close contacts of infected } \\
\text { cases }\end{array}$ & $\begin{array}{l}\text { Limitation in test harmonization and correlation } \\
\text { between different diagnostic methodologies }\end{array}$ \\
\hline $\begin{array}{l}\text { To verify the immune response in a studied } \\
\text { population }\end{array}$ & $\begin{array}{l}\text { Limited approvals by international leading health } \\
\text { institutions }\end{array}$ \\
\hline
\end{tabular}

$93 \%$ and negative predictive values of 74 to $92 \%$ [12]. These performance estimates were also observed in a meta-analysis of 17 studies with 1857 participants, with LFIAs exhibiting a pooled sensitivity of 66.0 (49.3 to 79.3) [13]. In keeping with other studies, the diagnostic performance of these tests reflected the duration of the illness with the worst performance observed in the first two weeks after symptom onset [12].

\section{The role of testing according to ASRM and ESHRE}

After the initial COVID-19 tsunami, and rapid cessation of routine clinical activity, many providers are now recommencing fertility care. However, our global professional bodies, the American Society for Reproductive Medicine (ASRM) and the European Society of Human Reproduction and Embryology (ESHRE), have provided discordant roadmaps for the resumption of clinical services. Despite the initial similarities for a questionnaire-based triage to identify infected staff and patients and recommendations for adoption of the now well-established generic infection control procedures [14-17], these guidelines exhibit little similarity concerning the deployment of SARS-CoV-2 testing strategies and their interpretation.

\section{SARS-CoV-2 testing according to ASRM}

The ASRM COVID Taskforce in their fourth update (published May 11, 2020), with subsequent confirmation in their fifth update (June 8, 2020), recognized that at present there is no sufficient information to recommend a specific algorithm or testing program in reproductive care (https://www.asrm. org/news-and-publications/covid-19/). Specifically, ASRM highlights that despite nucleic acid amplification test (NAAT)-based tests being the cornerstone of diagnostic testing for SARS-CoV-2, false-negative results can occur with inadequate sample collection or if the sample is collected early in the disease course and that the rapid tests which are increasingly being publicized may be less sensitive and specific. NAAT-based tests should, however, be considered preoperatively before reproductive surgery or other aerosol generating procedures, as part of the wider risk mitigation strategy. For antibody tests, ASRM recognized the considerable performance variability and stressed that the implications for immunity remain unclear. Furthermore, based on current evidence, antibody testing should not be used for patient or provider decision-making at this time and should not change adherence to guidelines for personal protective equipment use. ASRM conclude their guidance by encouraging its members to stay up-to-date with available tests and testing strategies as these continue to evolve.

\section{SARS-CoV-2 testing according to ESHRE}

The ESHRE's recommendations (https://www.eshre.eu/ Home/COVID19WG, published on May 5, 2020) are much more dependent on serological testing with proposed pathways incorporating their use for clinical decisionmaking (Fig. 1). In this algorithm, the ESHRE task force recommends that in the situation where either partner develops symptoms in the two weeks before ovarian stimulation, 


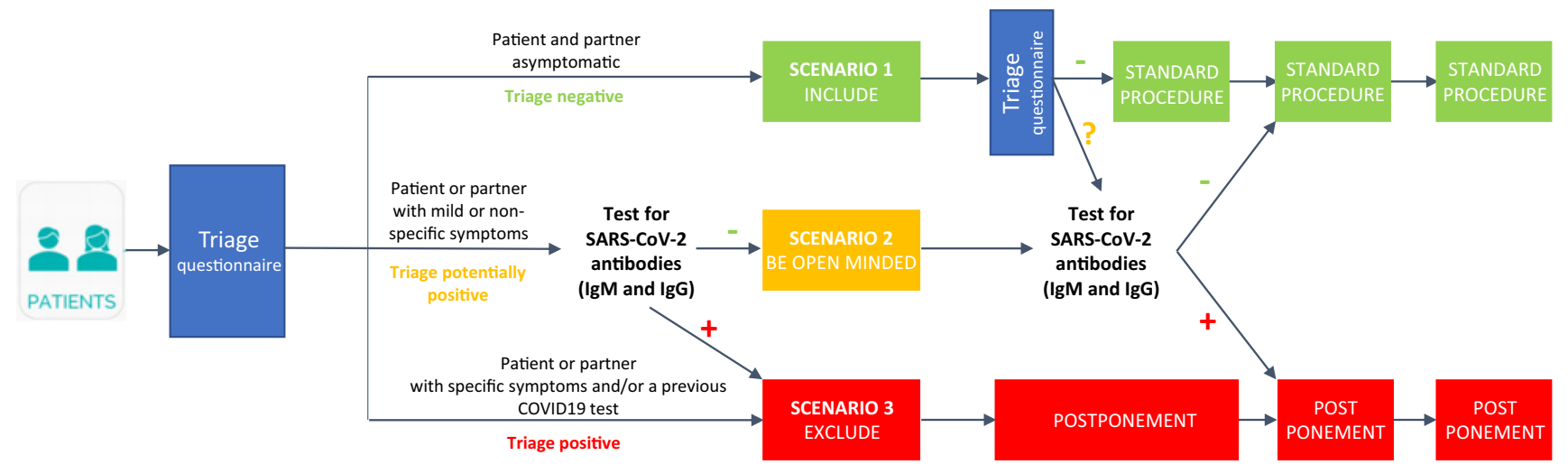

Fig. 1 The triage of patients as proposed by ESHRE

SARS-CoV-2 IgM/IgG testing may be used to decide to continue or postpone treatment, pending a negative or positive result respectively. Moreover, despite the initial concerns and a negative antibody test, there are further concerns during ovarian stimulation, and serological testing and/or NAATbased testing may be used again to decide whether to continue or not with the treatment.

The positions of the two main professional societies for human reproduction, therefore, appear divergent. While for ASRM testing for SARS-CoV-2 is currently not part of the routine workflow of infertility patients, ESHRE considers its use fundamental. So which approach should we adopt, and what are the risks associated with an inherent dependence on a testing algorithm?

\section{Is SARS-CoV-2 testing feasible in an IVF program?}

Before we implement routine SARS-CoV-2 testing within our IVF programs, several critical aspects need to be considered. These include whether testing within our clinics or by other testing facilities is viable and achievable. Secondly, whether results will be available promptly, accepting their known issues with sensitivity, specificity, and temporal nature. Finally, whether appropriate mitigation measures can be adopted depending on the result. A detailed assessment of all of these aspects is a prerequisite prior to the adoption of SARS-CoV-2 direct and serological tests into routine IVF care pathways.

The first hurdle for many will be the availability of testing. In a global pandemic, everyone is experiencing global pressure on supply chains for testing reagents and testing capacity infrastructure, and consequently, access to testing will differ between countries and even regions within a country. The fact that these resources may be managed by the government or national health service could further reduce individual availability of testing. Within the authors IVF institutions in Italy and UK, respectively, NAAT is not available within the IVF unit, rather SARS-CoV-2 testing is centralized, and symptomatic patients would be required to be referred to a community testing center and the NAAT result available potentially 24 to $48 \mathrm{~h}$ later. Serological testing is also subject to restrictions, with the tests not deployed for routine care of patients in NHS Scotland, due to the lack of clarity about the implications of a positive result in isolation $[6,10,13]$.

The suggested dependence on the NAAT to help stratify fertility patients does not reflect their potential for false negatives. Clinical management algorithms of COVID-19 incorporate repeat testing of multiple biological sites and management of the symptomatic patient using conventional pathways that assume that the patient is infected. Concerning serology, the antibody tests are not perceived as sufficiently accurate to be incorporated into diagnostic pathways in isolation or to triage patients with acute symptoms particularly in the early phases of infection $[6$, $10,13]$. Furthermore, although initial reports of antibody testing suggest good specificity, larger studies incorporating individuals with immunocompromised states or potential cross-reactivity from alternative coronaviruses or interference due to malignancy or autoimmune conditions are awaited. On this basis at present, serological testing is not to be considered useful as a primary diagnostic test for early acute infection, and rather should be considered as an effective supplement to NAAT [10].

From a patient's perspective, the combination of NAAT and antibody tests is optimal for accurate diagnosis throughout the broad spectrum of the disease. The NAAT exhibits very good sensitivity in the first few days after the onset of symptoms, whereas serological testing will perform poorly. In contrast, antibody detection will be particularly relevant for the later stages of infection where the virus has started to be or has been eliminated. The use of an isolated serological test, without the NAAT, may only really be relevant from a public health perspective when assessing overall SARS-CoV-2 exposure to facilitate accurate estimation of asymptomatic infections and provide an estimate of the real case count. 


\section{Conclusion}

The management of potentially infected SARS-CoV-2 infertility patients irrespective of where they are in the treatment pathway should be predicated on caution. Failure to fully appreciate the characteristics and limitations of the diagnostic tests may lead to disastrous consequences for the patient and the multidisciplinary team looking after them. The interpretation of a test for SARS-CoV-2 will depend on a combination of the accuracy of the test and the estimated risk of COVID-19 before performing the test. A positive test is strongly suggestive of infection due to its high specificity but moderate sensitivity, and the patient can be told with a high degree of confidence that exposure to the virus had occurred. Management should then be per local policies regarding positive cases. In contrast, negative tests need to be interpreted with caution, and a single negative SARS-CoV-2 test in a patient with suggestive symptoms should not be relied upon to exclude COVID-19. In this situation, it would still be safer for all concerned that the patient is treated as an infected individual, and local policies regarding retesting and isolation followed. With a global pandemic, global scientific collaboration is essential in the development of a robust evidence base. In our opinion, alignment of advice by both professional bodies would ensure that irrespective of geographical location patients and staff receive optimal care with minimal risk.

\section{Compliance with ethical standards}

Conflict of interest No funding bodies had any role in study design, data collection and analysis, decision to publish, or preparation of the manuscript. ALM has participated in Advisory Boards and received speakers and consultancy fees from Beckman Coulter, Gedeon Richter, Ferring, IBSA, Merck, MSD, Roche Diagnostics, and Theramex. SMN has participated in Advisory Boards and received speakers and consultancy fees from Access Fertility, Beckman Coulter, Ferring, Finox, Merck, MSD, Roche Diagnostics, and The Fertility Partnership.

Ethics approval Not applicable.

\section{References}

1. Zou L, Ruan F, Huang M, Liang L, Huang H, Hong Z, et al. SARSCoV-2 viral load in upper respiratory specimens of infected patients. N Engl J Med. 2020;382:1177-9.

2. To KK, Tsang OT, Leung WS, Tam AR, Wu TC, Lung DC, et al. Temporal profiles of viral load in posterior oropharyngeal saliva samples and serum antibody responses during infection by SARSCoV-2: an observational cohort study. Lancet Infect Dis. 2020. https://doi.org/10.1016/S1473-3099(20)30196.

3. He X, Lau EHY, Wu P, Deng X, Wang J, Hao X, et al. Temporal dynamics in viral shedding and transmissibility of COVID-19. Nat Med. 2020;26:672-5. https://doi.org/10.1038/s41591-020-0869-5.
4. Diao B, Wen K, Chen J, Liu Y, Yuan Z, Han C, Chen J, Pan Y, Chen L, Dan Y, Wang J , Chen Y, Deng G, Zhou H, Wu Y. Diagnosis of acute respiratory syndrome coronavirus 2 infection by detection of nucleocapsid protein. doi: https://doi.org/10.1101/ 2020.03.07.20032524

5. Tang YW, Schmitz JE, Persing DH, Stratton CW. The laboratory diagnosis of COVID-19 infection: current issues and challenges. J Clin Microbiol. 2020. https://doi.org/10.1128/JCM.00512-20.

6. Deeks JJ, Dinnes J, Takwoingi Y, et al. Antibody tests for identification of current and past infection with SARS-CoV-2. Cochrane Database Syst Rev. 2020;6:CD013652. Published 2020 Jun 25. https://doi.org/10.1002/14651858.CD013652.

7. Long QX, Liu BZ, Deng HJ, Wu GC, Deng K, Chen YK, et al. Antibody responses to SARS-CoV-2 in patients with COVID-19. Nat Med, 2020. https://doi.org/10.1038/s41591-020-0897-1.

8. Zhao J, Yuan Q, Wang H, Liu W, Liao X, Su Y, et al. Antibody responses to SARS-CoV-2 in patients of novel coronavirus disease 2019. Clin Infect Dis. 2020. https://doi.org/10.1093/cid/ciaa344.

9. Tang MS, Hock KG, Logsdon NM, Hayes JE, Gronowski AM, Anderson NW, et al. Clinical performance of two SARS-CoV-2 serologic assays. Clin Chem. 2020. https://doi.org/10.1093/ clinchem/hvaa120.

10. La Marca A, Capuzzo M, Paglia T, Roli L, Trenti T, Nelson S. Testing for SARS-CoV-2 (COVID-19): a systematic review and clinical guide to molecular and serological in-vitro diagnostic. RBMO. 2020. https://doi.org/10.1016/j.rbmo.2020.06.001.

11. González JM, Shelton WJ, Díaz-Vallejo M, Rodriguez-Castellanos VE, Zuluaga JDH, Chamorro DF, et al. Immunological assays for SARS-CoV-2: an analysis of available commercial tests to measure antigen and antibodies. MedRxiv. https://doi.org/10.1101/2020.03. 17.20037713

12. Lassaunière R, Frische A, Harboe Z, Nielsen A, Fomsgaard A, Krogfelt K, et al. Evaluation of nine commercial SARS-CoV-2 immunoassays. medRxiv. https://doi.org/10.1101/2020.04.09. 20056325.

13. Mayara LB, Gamuchirai T, Kunal AS, Campbell Jonathon R, Louis-Patrick H, Johnston James C, et al. Diagnostic accuracy of serological tests for covid-19: systematic review and meta-analysis. BMJ. 2020;370:m2516.

14. La Marca A, Niederberger C, Pellicer A, Nelson SM. COVID-19: lessons from the Italian reproductive medical experience. Fertil Steril. 2020;113:920-2.

15. Alviggi C, Esteves SC, Orvieto R, Conforti A, La Marca A, Fischer $\mathrm{R}$, et al. COVID-19 and assisted reproductive technology services: repercussions for patients and proposal for individualized clinical management. Reprod Biol Endocrinol. 2020;18:45. https://doi.org/ 10.1186/s12958-020-00605-z.

16. De Santis L, Anastasi A, Cimadomo D, Klinger FG, Licata E, Pisaturo V, et al. COVID-19: the perspective of Italian embryologists managing the IVF laboratory in pandemic emergency. Hum Reprod. 2020;35:1004-5. https://doi.org/10.1093/humrep/ deaa074.

17. Vaiarelli A, Bulletti C, Cimadomo D, Borini A, Alviggi C, Ajossa S, et al., COVID-19 and ART: the view of the Italian Society of Fertility and Sterility and Reproductive Medicine. Reprod Biomed Online. 2020 Apr 8.

Publisher's note Springer Nature remains neutral with regard to jurisdictional claims in published maps and institutional affiliations. 